\title{
3D-PTV measurement verified by UVP for unsteady and three-dimensional flow inside a suction sump
}

\author{
Katsuya HIRATA*, Atsushi SHINTANI*, Ryo KAWAGUCHI*, Katsuhisa INAGAKI*, \\ Takafumi NAGURA * and Tsuyoshi MAEDA* \\ *Department of Mechanical Engineering, Doshisha University, \\ Kyoto 610-0321, Japan \\ E-mail: khirata@mail.doshisha.ac.jp
}

Received 23 June 2015

\begin{abstract}
Our aim is to understand the complicated flow inside a suction sump in the vertical-wet-pit-pump configuration. The information of unsteady and three-dimensional flow structures is useful, not only for this complicated flow but also for other complicated flows which are commonly seen in various practical applications. To acquire such information, a three-dimensional particle tracking velocimetry (3D-PTV) technique is one solution. However, this technique includes more unknown factors in reliability and accuracy than other well-established measuring techniques. In the present study, we examine the simultaneous measurement using both of the 3D-PTV and another velocimetry, namely, an ultrasonic velocity profiler (UVP) with common tracer particles. This simultaneous measurement is expected to become an effective method to dissolve the above fatal defect of the 3D-PTV. As a result, we have successfully found out the suitable condition for the simultaneous measurement with high accuracy. Then, under this condition, we have revealed the time-mean and instantaneous velocity vectors of the unsteady and three-dimensional flow inside the suction sump.
\end{abstract}

Key words: Suction sump, Open sump, Three-dimensional flow, Unsteady flow, PTV, UVP, Pump, Two-phase flow

\section{Introduction}

Our aim is to understand the complicated flow inside a suction sump in the vertical-wet-pit-pump configuration. Most of the flows appearing in various industrial and environmental problems like the present suction-sump flow become often complicated owing to both of their unsteadiness with poor periodicity and their fully-three-dimensionality (Funaki et al., 2008, Hirata et al., 2009, Hirata et al., 2013a). So, in instantaneous and three-dimensional observations of such flows could give us useful and effective information.

In this context, a three-dimensional particle tracking velocimetry (hereinafter, referred to as 3D-PTV) is one of the potential solutions against such problems. The 3D-PTV is a technology to specify three-dimensional positions of tracer particles from a couple of stereo images, and to obtain the velocity vectors of tracer particles from their positions at different instants. The 3D-PTV has the following advantages: (1) there exists no sensor which disturbs flow; (2) we can get the information of many points at the same instant; (3) we can get three-dimensional information in the whole measurement volume. Furthermore, if we record the stereo images using a couple of synchronised video cameras with a high-speed frame rate, we can get a temporally-consecutive series of instantaneous and three-dimensional information of the whole flow. So, various 3D-PTV systems have been developed until now (Goldberg, 1985; Chang et al., 1985; Nishino et al., 1989; Adrian et al., 1991; Yamamoto et al., 1991; Doh et al., 2002).

In spite of the above advantages, the use of the 3D-PTV has been restricted. One of the main reasons exists in the difficulty to estimate its accuracy, which strongly depends upon (1) flow conditions, (2) tracer particles, (3) a lighting system and (4) a camera system, together with (5) a 3D-PTV's algorithm. Then, it seems difficult to establish the concrete and practical criteria for the 3D-PTV's accuracy in a general manner. So, at the present stage, it is rational to suppose that all the 3D-PTV measurements should be inevitably accompanied by the other verifying measurements 
using well-established techniques, to assure the 3D-PTV's accuracy.

In the present study, our concern is the complicated flow inside a suction sump in the vertical-wet-pit-pump configuration as is mentioned above. To acquire the information of such unsteady and three-dimensional flow, we examine the simultaneous measurement using both of the 3D-PTV and another velocimetry, namely, an ultrasonic velocity profiler (hereinafter, referred to as UVP) with common tracer particles. Specifically speaking, we investigate a possibility of the flexible and convenient measurement to check the 3D-PTV's accuracy, that is, the simultaneous measurement by the 3D-PTV with the UVP. The UVP has proposed and developed by Takeda (1986) and Takeda et al. (1992). This simultaneous measurement is expected to become an effective method to dissolve the above fatal defect of the 3D-PTV. In general, the UVP tends not to be applicable for a small number of large tracer particles. On the other hand, the 3D-PTV tends not to be applicable for a huge number of small tracer particles. We first carry out the simultaneous measurement using both of the 3D-PTV and the UVP of a simple swirling flow in a cylindrical container as a preliminary test. Once we found out the suitable conditions of tracer-particle concentration and size to conduct the simultaneous measurement using the 3D-PTV and the UVP, we could verify the 3D-PTV's accuracy easier and quicker than before. Afterwards, under the suitable conditions for the simultaneous measurement, we attempt to reveal the time-mean and instantaneous velocity vectors of the unsteady and three-dimensional flow inside the suction sump.

Of course, we may consider alternatives to the UVP as the verification measurements of the 3D-PTV, such as a hot-wire velocimetry (HWV), a laser-Doppler velocimetry (LDV), a two-dimensional or there-dimensional particle-image velocimetry (PIV) and a two-dimensional particle tracking velocimetry (2D-PTV). The HWV has high reliability and high accuracy, but it disturbs flow by its probe. The LDV has high responsibility and high accuracy, but it is often sensitive to the condition of tracer particles. As both of the HWV and the LDV give us the information at only one point in space, we are required to select the point carefully, especially for unknown complicated flows. For the LDV, the PIV and the UVP, we can consider a possibility to use the common tracer particles of the 3D-PTV, in order to neglect the complicated factors and to avoid the reduction of 3D-PTV's accuracy due to the intermingling tracer particles with various sizes. But, tracer-particle concentration should be kept high and homogeneous for the LDV. The PIV and the 2D-PTV give us neither the ping-point nor the one-dimensional information, but the two-dimensional information. However, they are not suitable for the verification measurements of the 3D-PTV, because of the use of similar kinds of source data such as flow-visualised photographs.

\section{Nomenclature}

\begin{tabular}{|c|c|}
\hline$B$ & : suction-sump breadth \\
\hline Bo & : Bond number or Eotvos number $\left(\equiv \rho g D^{2} / T\right)$ \\
\hline$d$ & : inside diameter of suction pipe \\
\hline$D$ & : outside diameter of a suction pipe \\
\hline$D_{N \mathrm{P}}$ & $:$ number density $=N_{\mathrm{P}}$ per a unit volume $\left[\mathrm{mm}^{-3}\right]$ \\
\hline$D_{N \mathrm{P}} V_{\mathrm{UVP}}$ & : $N_{\mathrm{P}}$ per a unit UVP-measurement volume \\
\hline$D_{\mathrm{P}}$ & : tracer particle diameter \\
\hline$F_{V P}$ & : volume fraction of tracer particles $=V_{\mathrm{P}}$ per a unit volume \\
\hline $\mathrm{Fr}$ & : Froude number $\left(\equiv U_{\mathrm{i}} /(g D)^{0.5}\right)$ \\
\hline$g$ & : gravitational acceleration $\quad\left[\mathrm{mm} / \mathrm{s}^{2}\right]$ \\
\hline$H$ & : water level \\
\hline$N_{\mathrm{P}}$ & : total number of tracer particles \\
\hline$Q$ & : (volumetric) flow rate \\
\hline $\operatorname{Re}$ & : Reynolds number $\left(\equiv \rho U_{\mathrm{i}} D / \mu\right)$ \\
\hline$S$ & : submergence \\
\hline$t$ & : time $\quad[\mathrm{s}]$ \\
\hline$T$ & : surface tension \\
\hline$U_{\mathrm{c}}$ & : channel-convection velocity $[\mathrm{mm} / \mathrm{s}]$ \\
\hline$U_{\mathrm{i}}$ & : suction-pipe-intake velocity $[\mathrm{mm} / \mathrm{s}]$ \\
\hline$v_{x}, v_{y}, v_{z}$ & : flow velocities \\
\hline$V_{\mathrm{P}}$ & : total volume of tracer particles \\
\hline$V_{\mathrm{UVP}}$ & : unit UVP-measurement volume \\
\hline
\end{tabular}




$\begin{array}{lll}\text { We } & : \text { Weber number }\left(\equiv U_{\mathrm{i}}(\rho D / T)^{0.5}\right) \\ x, y, z & : \text { coordinates } & {[\mathrm{mm}]} \\ X & : \text { back clearance } & {[\mathrm{mm}]} \\ Z & : \text { bottom clearance } & {[\mathrm{mm}]} \\ \mu & : \text { viscosity of fluid } & {[\mathrm{g} / \mathrm{mm} \mathrm{s}]} \\ \rho & : \text { density of fluid } & {\left[\mathrm{g} / \mathrm{mm}^{3}\right]} \\ \sigma & : \text { standard deviation } & \end{array}$

\section{Experimental Method}

\subsection{Model and experimental apparatus}

Figure 1 shows the present model, which is a simple system of a suction sump and a suction pipe with the vertical-wet-pit-pump configuration. Geometric parameters $D$ and $d$ are the outer and inner diameters of the suction pipe, respectively. The former is used as a characteristic length scale. The latter is fixed to $0.9 D$. The suction-pipe intake has a bell-mouth shape. - The diameter of the bell mouth is the same as $D$, having a simple geometry with less parameters. We should note that the influence of the bell mouth diameter upon the critical condition for the air entrainment is negligible according to Tagomori (1979), as $D<1.75 d$. - The pipe is placed vertically on the centre line of the suction sump. Three geometric parameters $B, X$ and $Z$ denote the breath of the suction sump, the clearance from the suction-pipe centre to the suction-sump back wall and the clearance between the suction-pipe intake and the suction-sump bottom wall, respectively. A geometric parameter $H$ is water level, namely, the height of a mean water-free surface, then the pipe's submergence depth $S$ is equal to $(H-Z)$. A characteristic velocity scale is the mean flow velocity $U_{\mathrm{i}}$ at the suction-pipe intake, which is defined by

$$
U_{\mathrm{i}}=4 Q /\left(\pi D^{2}\right)
$$

where $Q$ is inflow rate, or the flow rate into the suction pipe. We define the Froude number $F r$, the Reynolds number $R e$ and the Weber number We as follows.

$$
\begin{aligned}
& F r=U_{\mathrm{i}} /(g D)^{1 / 2} . \\
& R e=\rho U_{\mathrm{i}} D / \mu . \\
& W e=U_{\mathrm{i}}(\rho D / T)^{1 / 2} .
\end{aligned}
$$

Letters $g, \mu, \rho$ and $T$ denote the gravitational acceleration, fluid viscosity, fluid density and water-to-air surface tension, respectively. As a supplementary parameter, we define the Bond number (or The Eotvos) Bo as follows.

$$
B o=\rho g D^{2} / T \text {. }
$$

Table 1 summarises chief experimental parameters and their values in the main test of the present study, together with geometric and kinetic parameters in non-dimensional forms. These values are the same as those in one of the two test cases in Funaki et al. (2008) where not instantaneous but time-mean flow of the same system of a suction sump and a suction pipe as the present system is revealed by the UVP. Funaki et al. call the test case Case A. - For the details of the flow in Case A, see Subsection 4.3. -

Figure 1 also shows the present coordinate system. The origin $\mathrm{O}$ is on the suction-sump bottom wall and on the suction-pipe centre line. The $x$ axis is horizontal and parallel to the mean convection flow in the suction-sump channel, the $y$ axis is horizontal and normal to the mean convection flow, and the $z$ axis is vertical. We define three velocity components in the $x, y$ and $z$ directions as $v_{x}, v_{y}$ and $v_{z}$, respectively.

In the main test of the present study, an experimental apparatus is substantially identical with Funaki et al. (2008), Hirata et al. (2009) and Hirata et al. (2013a). A turbopump feeds working fluid (water) to a suction sump from a reservoir. We control the flow rate of the pump by a control valve, and then control the water level $H$ in the suction sump. In the upstream of the suction sump, we put a strainer to make flow uniform. A bend-type jet pump sends water 


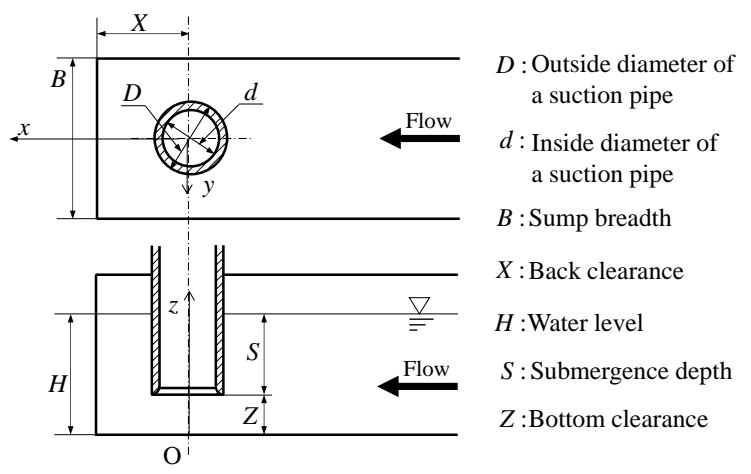

Fig. 1 Model: suction sump and suction pipe in the vertical-wet-pit-pump configuration, together with coordinate system.
Table 1 Experimental parameters.

\begin{tabular}{c|c}
\hline$D$ & $38 \mathrm{~mm}$ \\
\hline$(d)$ & $(34 \mathrm{~mm})$ \\
\hline$B / D$ & 3.16 \\
\hline$X / D$ & 1.58 \\
\hline$Z / D$ & 0.39 \\
\hline$S / D$ & 1.19 \\
\hline$U_{\mathrm{i}}$ & $0.6 \mathrm{~m} / \mathrm{s}$ \\
\hline$\left(U_{\mathrm{c}}\right)$ & $(0.095 \mathrm{~m} / \mathrm{s})$ \\
\hline$F r$ & 0.98 \\
\hline$R e$ & $2.2 \times 10^{4}$ \\
\hline$W e$ & 14 \\
\hline$(B o)$ & $(200)$ \\
\hline
\end{tabular}

up from the suction sump into the suction pipe, because the jet pump tends to induce less swirling than ordinary pumps. The jet pump itself is driven by another turbopump. Water from the suction pipe falls into the reservoir, then a water-circulation system is closed.

\subsection{D-PTV and UVP}

Figure 2 shows a schematic view of a close-up experimental apparatus near the suction pipe to explain the simultaneous measurement using both of the 3D-PTV and the UVP. A 3D-PTV system consists of the following: a YAG laser (No. 5 in the figure) with its power supply (No. 6) as a light source, a couple of high-speed video cameras (No. 4) with a frame rate of $1 / 500 \mathrm{~s}$, and a personal computer (No. 3) on which we conduct 3D-PTV analyses. The 3D-PTV requires more than two cameras which are synchronised with each other to take plural photographs at the same instant. We use a couple of cameras. As shown in Fig. 2, one camera is located outside the back wall of the suction sump, and the other camera is located outside the side wall of the suction sump. A UVP system consists of the following: an ultrasonic transducer (No. 7) as a UVP probe which is placed outside the suction sump, and a UVP monitor (No. 8) for the simultaneous measurement with the 3D-PTV.

In the present study, we use chemically-bridged polyethylene-resin spheres (its specific gravity is 0.92 ) with a mean diameter $D_{\mathrm{P}}$ as the common tracer particles. The tracer particles are coated with fluorescent paint, which are illuminated by the YAG laser. Actual tracer particle diameter $D_{\mathrm{P}}$ is controlled by the thickness of the paint's layer. Table 2 shows four kinds of tested conditions Conditions I-IV of the tracer particle. Parameters $V_{\mathrm{P}}, F_{V \mathrm{P}}, N_{\mathrm{P}}, D_{N \mathrm{P}}$, and $V_{\mathrm{UVP}}$ are the total volume of tracer particles, the volume fraction of tracer particles (or the volume of tracer particles per a unit volume), the total number of tracer particle, the number density (or the number of tracer particles per a unit volume) and the unit volume of the UVP measurement, respectively. Conditions I- III have the same $D_{\mathrm{P}}$ and different $D_{N \mathrm{P}}$. Condition IV has different $D_{\mathrm{P}}$ from the conditions I-III, but has almost the same $D_{N \mathrm{P}}$ as Condition III. By a preliminary test which will be described later, we attempt to find out the suitable condition for the simultaneous measurement among the four conditions.

The details of the 3D-PTV measurement is as follows (also, see Funaki et al. (2009) and Hirata et al. (2013b)). The 3D-PTV system is calibrated in advance, then we take a couple of stereo images of a measurement volume using two high-speed video cameras with a frame rate of $1 / 500 \mathrm{~s}$ as shown in Fig. 2. Total recording time is $0.6 \mathrm{~s}$, which represents 300 frames. This total recording time might not be enough for the flow (see Subsection 4.3), because of the restriction of the 3D-PTV system like the equipped memory size. Each image adequately covers the measurement volume. Next, we calculate the three-dimensional positions of tracer particles from couple of stereo images. The velocity vectors of tracer particles are determined from two consecutive information of the tracer-particle positions. All data processings have been done in a personal computer. Details is as follows.

For the 3D-PTV calibration, first, we fix two cameras so that their fields of view just cover up the whole measurement volume. If possible, two camera centers should cross, and should be right-angled with each other, to 
attain higher accuracy. Second, we set as many datum points as possible in the measurement volume, and take a couple of stereo photographs. The three-dimensional positions of these datum points are known in advance. In the present study, the number of the datum points is 43 . Thirdly, we can determine a coordinate system, by which we construct a virtual space corresponding to the real space in the personal computer.

For the specification of the tracer-particle positions in three dimensions, we use four consecutive series of informations. First, we specify the centre positions of tracer-particles on each two-dimensional photograph, through a digital image processing. Namely, after the removal of the background image which is the ensemble mean of stationary pixels during all the measuring time, we consider the maximum luminosity position as the tracer-particle centre. In order to identify each tracer particle from surrounding tracer particles, we suppose two thresholds, that is, the minimum size of the tracer particle and the minimum luminosity for the tracer particle. These thresholds can make the identification more accurate, but we should specify appropriate values depending on experimental conditions. Second, we determine the straight line on which there exist both a tracer-particle centre and a camera's view point. Then, we can see that a tracer-particle position is decided on the coordinate system as an intersection of the two straight lines from a couple of stereo images. Now, we know all the tracer-particle positions in the real 3D space on each time step. For the identification of the same tracer particle on different time steps, a genetic algorithm is used, based on successive four time steps' data. So, we can get tracer-particle velocity vectors which are obtained from successive two time steps' data.

The details of the UVP measurement is as follows (also, see Funaki et al. (2008)). Using the UVP, we can get fine-time-resolution informations, which are not merely the time histories of velocity vectors on a few spatially-fixed points like HWV and LDV. That is, we can get instantaneous velocity profiles by the UVP in terms of the Doppler effect of ultrasonic echoes. Specifically speaking, the UVP is a velocity-measuring method utilising the Doppler frequency shift of echo signals of pulsative ultrasonic waves reflected on minute tracer particles in fluid, and simultaneously a distance-measuring method utilising the time lag between emitted and returned waves. Then, we can get a simultaneous velocity profile on a line, even in opaque fluids such as liquid metals. Furthermore, the UVP has an advantage on accuracy in comparison with the PTV and the PIV, and does not require clearly-visualised photographs.

In the present study, we use a UVP of UVP X-2-PS by Met Flow SA with a frequency of $4 \mathrm{MHz}$. The number of measuring points is 128 in one profile, and then, the space resolution on the profile is $0.75 \mathrm{~mm}$. As the diameter of the ultrasonic beam is $5 \mathrm{~mm}$, one measuring volume is a disc with a diameter of $5 \mathrm{~mm}$ and with a thickness of $0.75 \mathrm{~mm}$. We get consecutive 1024 profiles in each measurement with an interval of $32 \mathrm{~ms}$ or more.

We should note that the UVP enables us to know only one component of velocity vectors, which is parallel to the axis of the UVP probe an ultrasonic transducer. Moreover, the spatial range for the measurement is restricted, as the position of the UVP probe is outside of the suction sump.

To make sure the effectivity of the present measurement further, we conduct another measurement using a still camera with a shutter speed of $1 / 20 \mathrm{~s}$ as a conventional technique with high reliability and low accuracy, in addition to the simultaneous measurement with the UVP. - By the still-camera-measurement technique, we visualise path lines (paths), namely, time integrals with low accuracy and high reliability. Smaller data-process number of this technique than other technique brings us high reliability in addition to the robustness due to the time integrals. - In the still-camera measurement, we can know a flow-velocity vector from both the length and direction of the short path line of each tracer particle, which is specified on the basis of a photograph with an adequate exposure time.

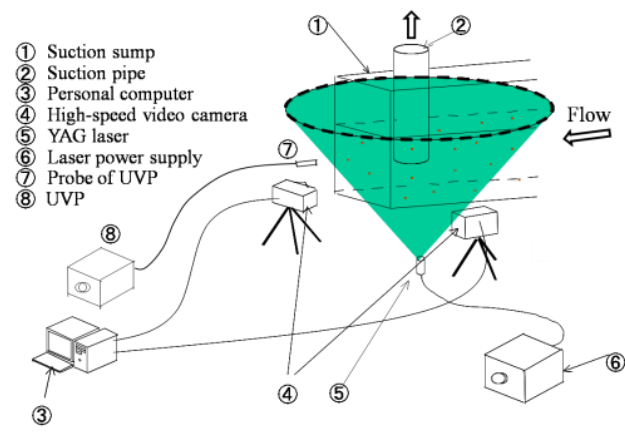

Fig. 2 Schematic view of a close-up experimental apparatus near a suction pipe for simultaneous measurement using both of 3D-PTV and UVP.

Table 2. Conditions of tracer particle.

\begin{tabular}{|l|l|l|l|l|}
\hline Condition & $\mathrm{I}$ & $\mathrm{II}$ & $\mathrm{III}$ & $\mathrm{IV}$ \\
\hline$D_{\mathrm{p}}[\mathrm{mm}]$ & $0.6-1.2$ & $0.6-1.2$ & $0.6-1.2$ & $0.3-0.6$ \\
\hline$V_{\mathrm{P}}\left[\mathrm{mm}^{3}\right]$ & 930 & 1900 & 3700 & 440 \\
\hline$F_{\mathrm{VP}}[\%]$ & 0.05 & 0.11 & 0.2 & 0.03 \\
\hline$N_{\mathrm{P}}$ & 2500 & 5000 & 10000 & 9300 \\
\hline$D_{N \mathrm{P}}\left[\mathrm{mm}^{-3}\right]$ & 0.001 & 0.003 & 0.006 & 0.005 \\
\hline$D_{N \mathrm{P}} V_{\mathrm{UVP}}[-]$ & 0.041 & 0.083 & 0.17 & 0.15 \\
\hline
\end{tabular}




\subsection{Preliminary test for tracer-particle condition}

As a preliminary test for the simultaneous measurement using both of the 3D-PTV and the UVP, we introduce a simple swirling flow, which is one of three-dimensional turbulent flows. Figure 3 shows a schematic view of the experimental apparatus in this preliminary test. A cylindrical container (No. 1 in the figure) made of transparent acrylic resin is filled with water. We put the cylindrical container on a magnetic stirrer (No. 2). A stirring object (No. 3) on the cylindrical-container bottom drives the swirling flow. A 3D-PTV system (Nos. 4-7) and a UVP system (Nos. 8 \& 9) are the same as these in the main test shown in Fig. 2. For accurate 3D-PTV measurements in the preliminary test, we have to diminish the image's distortion due to the curvature of a cylindrical-container surface. Then, we put a rectangular-prism container which covers the cylindrical container, and fill the space between both the containers with water as well.

Figure 4 shows the coordinate system in the preliminary test, with the position of the UVP probe. We put the probe between the rectangular-prism and cylindrical containers. On the other hand, one camera of the 3D-PTV system is located above the container, and the other camera is located in front of the container, as shown in Fig. 3 . Figure 5 shows sample stereo photographs by both of the cameras. The tracer-particle condition is close to Condition II. While most of fluid is in a swirling motion, we can confirm that tracer particles are homogeneously scattered being suspended in fluid.

\section{Results and Discussion}

\subsection{Preliminary test for simultaneous measurement using common tracer particles}

Figure 6 shows the raw results in four Conditions I-IV in the preliminary tests. More specifically, each figure denotes the time history of flow velocity $v_{y}$ detected by the UVP in each condition at $y=0 \mathrm{~mm}$. The ordinate is

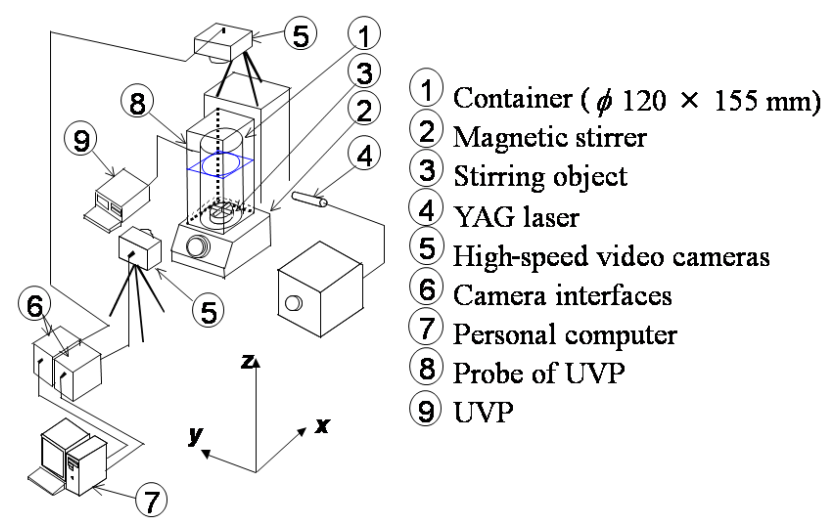

Fig. 3 Schematic view of experimental apparatus in preliminary test.

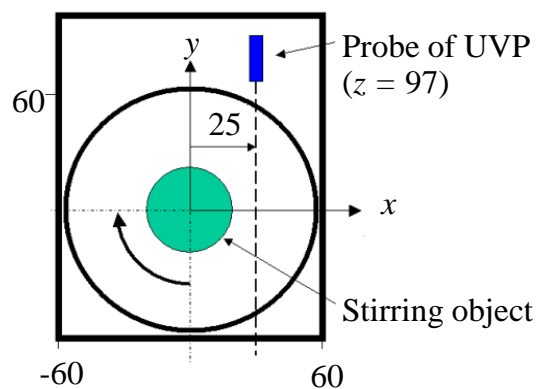

Fig. 4 Top view of a container in preliminary test, together with coordinate system and the position of a UVP probe (unit: $\mathrm{mm}$ ).

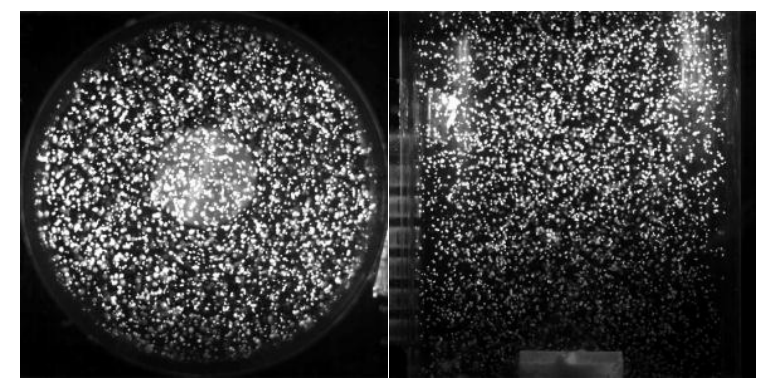

$\begin{array}{ll}\text { (a) Top view } & \text { (b) Side view }\end{array}$

Fig. 5 Sample stereo photographs: top and side views of a container in preliminary test. 
normalised $v_{y}$ using its time-mean value $\overline{v_{y}}$. In Condition I, we can see scattering data between 0 and 2 in $v_{y} / \overline{v_{y}}$ (see figure (a)). Then, the standard deviation $\sigma$ becomes such a large value as 0.49 . The data at $v_{y} / \overline{v_{y}} \leq 0.5$ is considered to be the error due to the lack of tracer particles in the present UVP-measurement volume. As the number density $D_{N P}$, namely, the number of tracer particles per a unit volume increases, the values of $v_{y}$ concentrate into their mean value $\overline{v_{y}}$ with a small $\sigma=0.25$ (see Condition II in figure (b)). While there exist some data with $v_{y} / \overline{v_{y}} \approx 0$ due to the lack of tracer particles in the UVP-measure volume even in Condition II, the total number of the data with $v_{y} / \overline{v_{y}} \approx 0$ is relatively sparse. Therefore, such a effect is negligible on the time-mean value $\overline{v_{y}}$. Again, we can confirm that Condition III (in figure (c)) is in good accuracy, as well as Condition II with a small amount of the error data with $v_{y} /$ $\overline{v_{y}} \approx 0$ which are not shown in figure (c). So, for precise UVP measurements, we need that $D_{N \mathrm{P}}$ is greater than the value in Condition II. Next, concerning the size of tracer particle, we compare Conditions III (in figure (c)) and IV (in figure (d)). The particle diameter $D_{\mathrm{P}}$ in the condition III is almost two-times bigger than that in Condition IV. To conclude, we cannot see any effective size effects of the tracer particles. And, all the other tested particle diameter $D_{\mathrm{P}}$ could be in a suitable range for the UVP measurement.

Figure 7 shows the comparison between the 3D-PTV and the UVP in the preliminary test, concerning the spatial profile of time-mean velocity $\overline{v_{y}}$. Figures (a) and (b) represent to Conditions I and II, respectively. In Condition I, $v_{y}$ obtained by the UVP is lower than that by the 3D-PTV. As is shown in Fig. 6(a), the UVP data include many errors. Then, the UVP data in Fig. 7(a) are considered to become lower than the actual values. On the other hand, in Fig. 7(b), the 3D-PTV data are in good agreement with the UVP ones. So, in Condition II, it is considered to have successfully conducted accurate measurements by the 3D-PTV, and the referring UVP data are reliable as shown in Fig. 6. Then, larger $D_{N P}$ than Condition II is considered to be suitable for the simultaneous measurement using both of the 3D-PTV and the UVP. On the other hand, we have also confirmed that the 3D-PTV measurements using much larger $D_{N P}$ than Condition II easily induce the reduction of $\overline{v_{y}}$ due to the failures in particle identification, in addition to the increase of numerical processes. To conclude, in the preliminary test, we have found that a suitable condition for the simultaneous measurement can be realised in Condition II where $D_{\mathrm{P}}=0.6-1.2 \mathrm{~mm}$ and $D_{N \mathrm{P}}=0.083 \mathrm{~mm}^{-3}$.

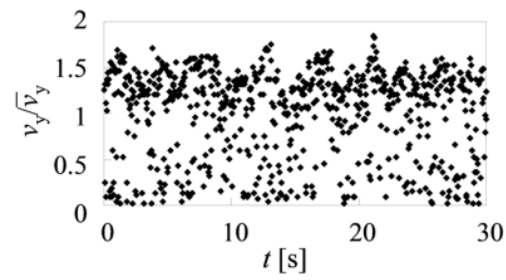

(a) Condition I (standard deviation $\sigma=0.49$ )

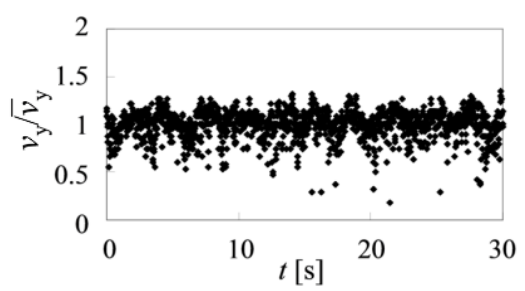

(c) Condition III (standard deviation $\sigma=0.16$

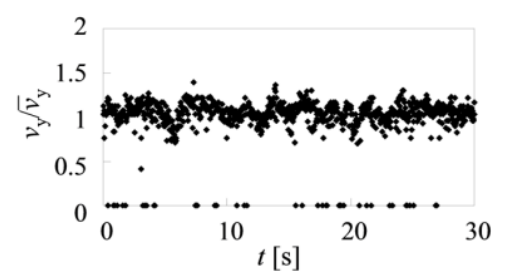

(b) Condition II (standard deviation $\sigma=0.25$ )

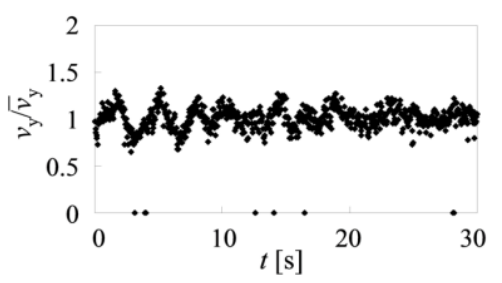

(d) Condition IV (standard deviation $\sigma=0.15$ )

Fig. 6 Time history of flow velocity by UVP at $y=0 \mathrm{~mm}$ in preliminary test.

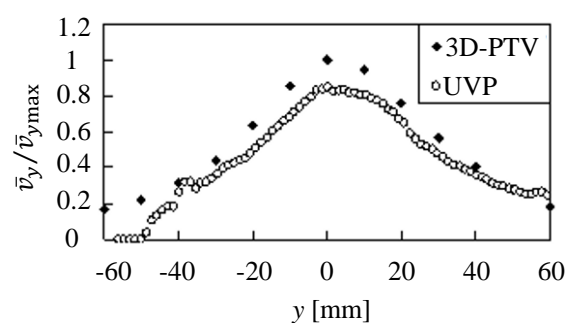

(a) Condition I

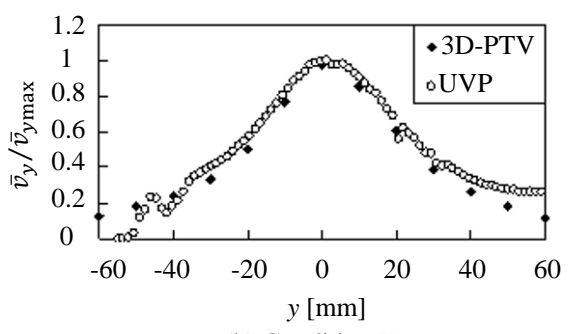

(b) Condition II

Fig. 7 Profile of time-mean velocity $\overline{v_{y}}$ in preliminary test. 


\subsection{Uniform flow in suction sump}

We first conduct the simultaneous measurement using both of the 3D-PTV and the UVP on a simple flow in the actual suction sump in the main test. As the simple flow, we regard an almost uniform flow everywhere in the whole measurement volume, which is introduced by forcedly controlling the channel of the suction sump with an attached guide wall as shown in Fig. 8. That is, the figure shows the schematic top view of the experimental apparatus with the controlled channel for uniform flow, where a channel breadth $B=100 \mathrm{~mm}$, a water level $H=100 \mathrm{~mm}$ and a channel-convection velocity $U_{\mathrm{c}}=110 \mathrm{~mm} / \mathrm{s}$. Exactly speaking, $U_{\mathrm{c}}$ represents the timely-and-spacially-averaged velocity vectors in the controlled channel. The suction pipe is offset to improve the uniformity of the controlled channel flow. The arrangements and set-ups of the 3D-PTV and the UVP are the same as Fig. 2.

Figure 9 shows an example of the velocity profile by the simultaneous measurement using both the 3D-PTV and the UVP in the controlled channel for uniform flow at $y^{\prime} / B=0$ and $z / H=0.40$, where $y^{\prime}$ denotes an auxiliary coordinate of $y$ with its origin at the controlled-channel centre. - The profiles are time-mean ones to be exact, while the instantaneous velocity is almost identical to the time-mean velocity. Because, the flow is approximately steady in the controlled channel. - We can see good agreement between the 3D-PTV and the UVP.

Figure 10 shows two examples of velocity vectors by 3D-PTV in the controlled channel for uniform flow. More specifically, figures (a) and (b) represent the flow-velocity vectors inter-polated with an equivalent separation in space on the $x$-z plane at $y^{\prime} / B=0$ and on the $x-y^{\prime}$ plane at $z / H=0.40$, respectively. They are time-mean ones, as well. We can see that the flow is almost uniform. This also assures the reliability of the present 3D-PTV.

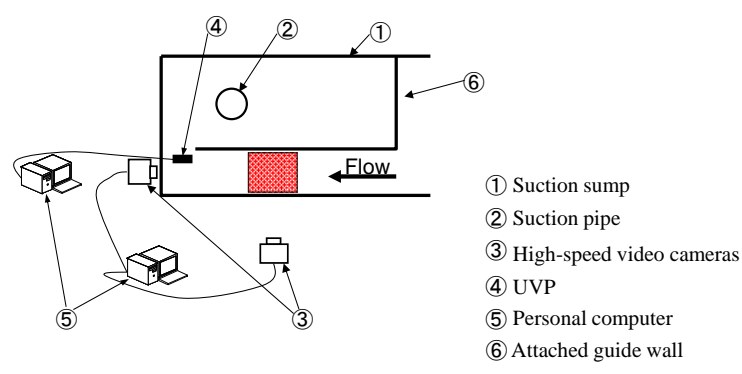

Fig. 8 Schematic top view of an experimental apparatus for simultaneous measurement using both of 3D-PTV and UVP in a controlled channel for uniform flow $\left(B=100 \mathrm{~mm}, H=100 \mathrm{~mm}\right.$ and $\left.U_{\mathrm{c}}=110 \mathrm{~mm} / \mathrm{s}\right)$.

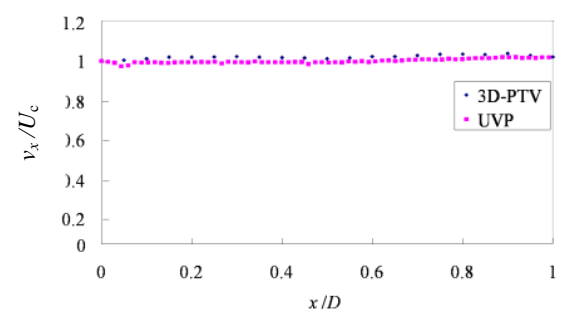

Fig. 9 Velocity profile by simultaneous measurement using both of 3D-PTV and UVP in a controlled channel for uniform flow, at $y^{\prime} / B=0$ and $z / H=0.40$.

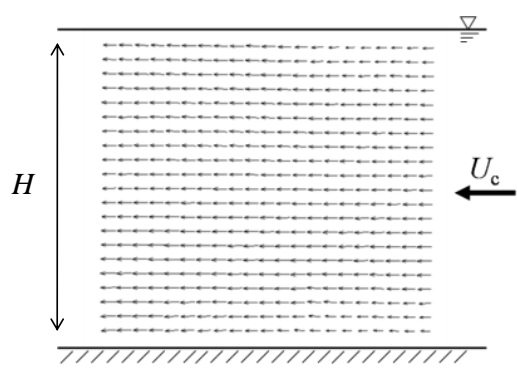

(a) On the $x$ - $z$ plane at $y^{\prime} / B=0$

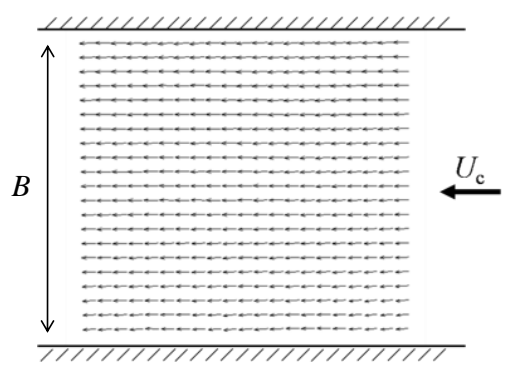

(b) On the $x$-y' plane at $z / H=0.40$

Fig. 10 Velocity vectors by 3D-PTV in a controlled channel for uniform flow. 


\subsection{Time-mean and instantaneous flow in suction sump}

Figure 11 shows an example of the simultaneous measurement using both of the 3D-PTV and the UVP on the complicated flow in the actual suction sump in the main test, together with the measurement using a still camera as a conventional technique with high reliability and low accuracy. More specifically, the figure shows the profile of the $x$-component of time-mean flow-velocity vector averaging over $0.6 \mathrm{~s}$ at $y / D=0.79$ and $z / D=1.05$. To be exact, the averaging time in the still-camera measurement corresponds to $1 / 20 \mathrm{~s}$, which is much shorter than the 3D-PTV and the UVP. To conclude, we can see good agreement from a qualitative viewpoint among the 3D-PTV, the UVP and the still-camera measurement despite the complexity of the flow. From a quantitative viewpoint, we can confirm that the average of the relative error of $\bar{v}_{x}$ by 3D-PTV to the UVP is $13 \%$, although the relative error locally attains $24 \%$ at $x / D \approx-0.5$.

Concerning the averaging number and the averaging time to obtain the time-mean velocity vectors, Funaki et al. (2008) has shown in the UVP measurement on the same flow as the present study that a 200-averaged result is in good agreement with a 500-averaged one, while a 50-averaged one differs from a 500-averaged one especially in the downstream of the suction pipe (at $x / D>0$ ). So, Funaki et al. have concluded that the averaging number of 200 is enough. The averaging time corresponding to this averaging number of 200 is equal to $6.4 \mathrm{~s}$. In the present study, we conduct each 3D-PTV measurement with an averaging number of 300 and an averaging time of $0.6 \mathrm{~s}$, and each UVP measurement with an averaging number of about 10 and an averaging time of $0.6 \mathrm{~s}$ corresponding to a measuring interval of $65 \mathrm{~ms}$. In the still-camera measurement, the corresponding averaging number and time are 1 and $1 / 20 \mathrm{~s}$, respectively. While the averaging time of the 3D-PTV will be further examined at least, this seems not fatal to discuss the present flow from a qualitative point of view, because the present flow is almost steady without large perturbations (as will be mentioned below) especially in the upstream of the suction pipe (at $x / D \leq 0$ ) where perturbations are much smaller (as will be shown in Figs. 12 and 18). The still-camera measurement is conducted inconsiderably in the upstream of the suction pipe.

Now, we have acquired the reliability and the accuracy in measurements by the 3D-PTV in the present suction sump. So, we will reveal the flow next by the 3D-PTV measurement. Figure 12 shows the time-mean flow obtained by the 3D-PTV. Specifically speaking, the figure denotes the projections on the $y-z$ plane of the time-mean velocity vectors in six cross sections. Figures (a), (b), (c), (d), (e) and (f) are at $x / D=-1.32,-0.79,-0.26,0.26,0.79$ and 1.32 , respectively.

As was mentioned in Subsection 3.1, the present flow is the same as that in one of the two test cases in Funaki et al., which is called Case A. In Case A, we often observe the air entrainment from the free surface into the suction pipe. More specifically, we almost every instants, there stably exist a pair of symmetric air strings (or string-like air bulks) from the free surface into the suction-pipe intake in the suction sump, whose positions are in the downstream of the suction pipe. Owing to small perturbations, the symmetry is occasionally broken. At such an instant, one or none air string appears instead of the symmetric twin air strings.

Funaki et al. have summarised their results based on the UVP measurement in a schematic diagram, which represent the time-mean and three-dimensional flow structure near the suction pipe in the same flow as the present study. That is, the flow is symmetric about the vertical centre plane of the suction sump. In the downstream of the suction pipe, there exist two vortex filaments A-3 and A-4 with large-magnitude vorticities. One end of each vortex filament reaches the free surface, and the other end reaches the suction-pipe intake. These vortex filaments usually accompany the air strings, which correspond to the air cores by the air entrainment into the suction-pipe intake. In the upstream of the suction pipe, there exist a pair of vortex filaments A-1 and A-2 with opposite rotations, whose axes are longitudinal.

In Fig. 12, the flows in the upstream and in the vicinity of the suction pipe at $x / D \leq 0.26$ (in figures (a) - (d)) resemble one another. In other words, the fluid at $y / D>0$ tends to concentrate toward the centre on the suction-sump bottom from the centre near the free surface with a clockwise turning motion. On the other hand, the fluid at $y / D<0$ tends to concentrate toward the centre on the bottom from the centre near the free surface as well, but with an anticlockwise turning motion. Then, we can recognise the symmetric flow about the vertical centre line at $y / D=0$, in each figure. These two symmetric turning motions are consistent with the vortex filaments A-1 and A-2 which are suggested on the basis of the UVP measurement by Funaki et al. Besides, we clearly see that a remarkable converging point of flow appears near the centre of the suction-sump bottom in each figure. On the other hand, we clearly see no diverging points, because of the existence of the suction pipe in figures (c) and (d), and because of very-small values of 
the magnitude $\left(v_{y}{ }^{2}+v_{z}{ }^{2}\right)^{1 / 2}$ of the projected velocity vector in figures (a) and (b) where we see ambiguous diverging/saddle points in an area at $y / D \approx 0$ and $z / D \approx 1$ instead of one clear diverging point. Of course, the very small magnitude in the area is consistent, because the mainstream component $v_{x}$ in the $x$ direction is much more dominant than the others almost everywhere. Then, it tends to induce the symmetry's breaking in the area.

Again in Fig. 12, the flows in the downstream of the suction pipe at $x / D>0.26$ (in figures (e) and (f)) resemble each other, as well. In other words, we cannot see one clear converging point in contrast with figures (a) - (d). The fluid at $y / D>0$ is almost in a clockwise circulating motion with a centre at $y / D \approx 0.5$ and $z / D \approx 1$. On the other hand, the fluid at $y / D<0$ is almost in an anticlockwise circulating motion with a centre at $y / D \approx-0.5$ and $z / D \approx 1$. Then, we can recognise the symmetry of flow about the vertical centre line at $y / D=0$, in each figure. These twin symmetric circulating motions are consistent with the vortex filaments A-3 and A-4 by Funaki et al., as well. Besides, we clearly see an upward streaming at $y / D \approx 0$, in contrast with figures (a) and (b) where we see a rather downward streaming due to the existences of the remarkable converging point near the bottom and the ambiguous diverging/saddle-points area near the centre.

Figure 13 shows time-mean velocity vectors by the UVP on the $y-z$ plane at $x / D=0.63$ (Funaki et al., 2008). Figure 14 shows the paths of tracer particles by a still camera on the $y-z$ plane at $x / D=0$, with a shutter speed of $1 / 20 \mathrm{~s}$. $-\mathrm{Of}$ course, the tracer-particle condition is much different from Condition II. - As these figures well correspond to Fig. 12 in spite of much different averaging times from Figs. 13 and 14, we can again confirm the reliability and the accuracy of the present 3D-PTV measurement.

As well, we compare the time-mean flow in the $x-z$ plane, instead of the $y-z$ plane. Figures 15,16 and 17 show time-mean velocity vectors by the $3 \mathrm{D}-\mathrm{PTV}$ at $y / D=-0.66$, time-mean velocity vectors by the UVP at $y / D=-0.63$ (Funaki et al., 2008), and the paths of tracer particles by a still cameras at $y / D=0.79$. We can see that the flows in these figures resemble with one another, that is, there exist (1) a downward streaming at $x / D \approx 0.5$ and (2) a clockwise circulating motion with a centre at $x / D \approx 0.5$. Then, we can confirm the reliability and the accuracy of the present 3D-PTV measurement, three times.

From such an observation as the air strings in the present flow are stable, we can anticipate that the present flow is stable as well. In fact, the instantaneous flows obtained by the 3D-PTV at most of measuring instants are similar with the time-mean one shown in Fig. 12. On the other hand, we occasionally observe that the symmetry of a pair of the air strings is broken with poor periodicity. At such instants, the instantaneous flow might be different from the time-mean one. Figure 18 is an example at the instants, where only one air string appears at $x / D \approx 0.8$ and $y / D \approx 1.2$. Fortunately, there rarely exist air bubbles which make 3D-PTV's accuracy worse. - Specifically speaking, the figure shows the projections on the $y-z$ plane of the instantaneous velocity vectors in six cross sections at $x / D=-1.32$ (in figure (a)), -0.79 (in figure (b)), -0.26 (in figure (c)), 0.26 (in figure (d)), 0.79 (in figure (e)) and 1.32 (in figure (f))), corresponding to the time-mean velocity vectors in Fig. 12.

In Fig. 18, the flows in the upstream and in the vicinity of the suction pipe at $x / D \leq 0.26$ (in figures (a) - (d)) resemble one another. Besides, the flow is almost the same as Fig. 12. In other words, we can see (1) the two symmetric turning motions being consistent with the vortex filaments A-1 and A-2, (2) one remarkable converging point of flow near the centre on the suction-sump bottom, and (3) ambiguous diverging/saddle points with very-small magnitudes in an area at $y / D \approx 0$ and $z / D \approx 0$. On the other hand, the flows in the downstream of the suction pipe at $x / D>0.26$ (in figures (e) and (f)) do not resemble each other, except for a common downward streaming from the free surface at $y / D$ $\approx 1$. The common downward streaming could be affected by the air string which occasionally appears at the instant at $x / D \approx 0.8$ and $y / D \approx 1.2$. Besides, either of both the figures does not resemble Figs. 12(e) and 12(f). Specifically speaking, in Fig. 18(e), we cannot see neither (1) the symmetric twin circulating motion nor (2) the upward streaming near the suction-sump centre (at $y / D \approx 0$ ). In contrast, in Fig. $18(\mathrm{e})$, we see a rather downward streaming at $y / D \approx 0$ due to a converging point near the bottom centre and ambiguous diverging/saddle points in an area near the surface. This is similar with Figs. 12(a)-(d). But in a strict sense, the downward streaming is not vertical but inclined, since the diverging/saddle area is far from the suction-sump centre. In Fig. 18(f), the flow resembles neither one in Fig. 18(e) nor the others in Figs. 12 and 18. In other words, the flow is governed by a single circulating motion with a centre at $y / D \approx$ 0.3 and $z / D \approx 1$. In summary, the instantaneous flow in the downstream of the suction pipe tends to fluctuate much more with complicated flow structures than the flow in the upstream and in the vicinity of the suction pipe which is similar with the time-mean flow at any time. 


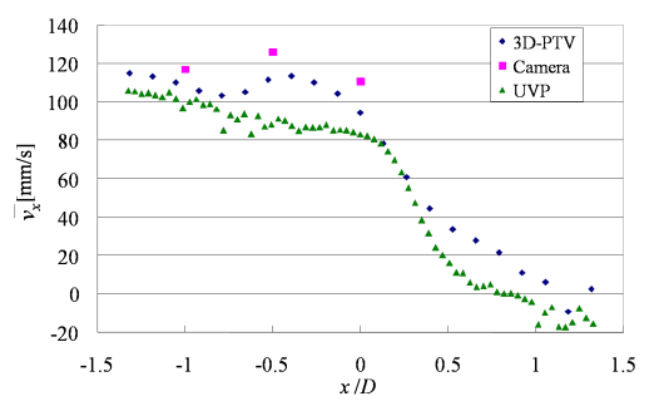

Fig. 11 Time-mean velocity profile at $y / D=0.79$ and $z / D=1.05$.

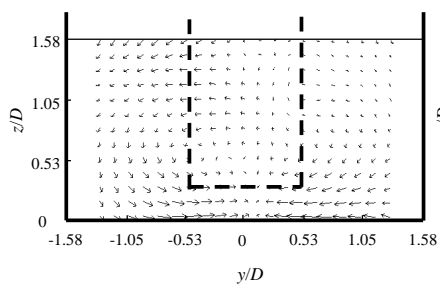

(a) $x / D=-1.32$.

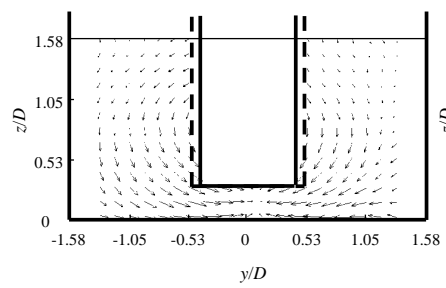

(d) $x / D=0.26$.

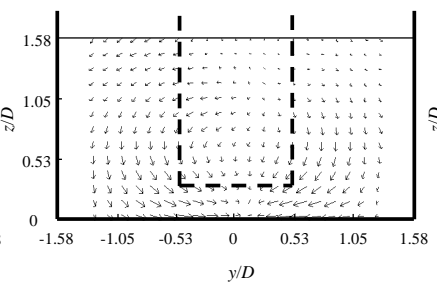

(b) $x / D=-0.79$.

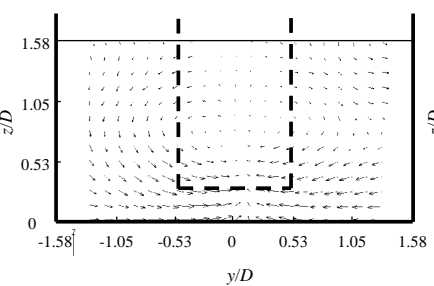

(e) $x / D=0.79$.

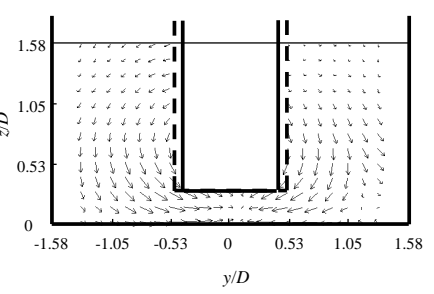

(c) $x / D=-0.26$.

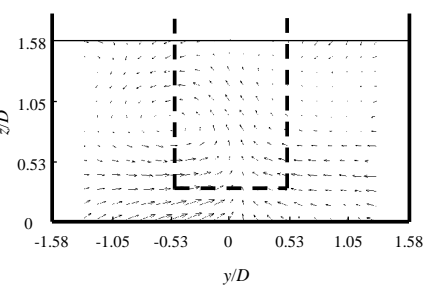

(f) $x / D=1.32$.

Fig. 12 Time-mean velocity vectors by 3D-PTV.

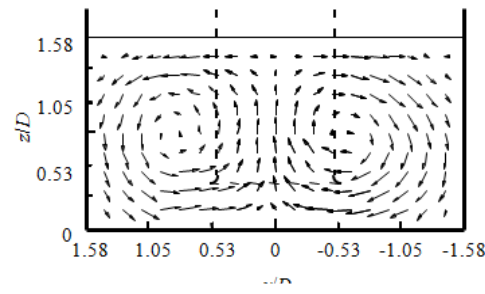

Fig. 13 Time-mean velocity vectors by UVP at $x / D$ $=0.63$ (Funaki et al., 2008).

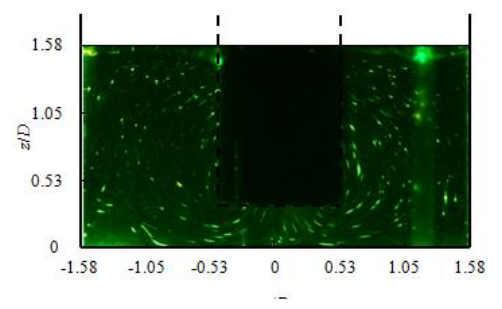

Fig. 14 Paths of tracer particles by a still camera on the $y-z$ plane at $x / D=0$, with a shutter speed of $1 / 20 \mathrm{~s}$. 


\section{Conclusion}

In order to understand the complicated flow inside a suction sump in the vertical-wet-pit-pump configuration, we have conducted the measurement by three-dimensional particle tracking velocimetry (3D-PTV) technique. As the technique includes more unknown factors in reliability and accuracy than other established measuring techniques, we have introduced the simultaneous measurement by the 3D-PTV with another velocimetry, namely, an ultrasonic velocity profiler (UVP) with common tracer particles. As a result, we have successfully found out the suitable condition (Condition II) for the simultaneous measurement with high accuracy. Then, under this condition, we have revealed the time-mean and instantaneous velocity vectors of the unsteady and three-dimensional flow using the 3D-PTV verified by the UVP in addition to the conventional still-camera measurement. The instantaneous flow in the downstream of the suction pipe tends to fluctuate much more with complicated flow structures than the flow in the upstream and in the vicinity of the suction pipe which is similar with the time-mean flow at any time. Even in other cases apart from the present flow, the simultaneous measurement with the UVP could be one of the reinforcements of the useful and powerful 3D-PTV applications especially in reliability and accuracy, while we should note that the suitable condition will be possibly modified depending upon flow velocity, fluid density, fluid transparency and so on.

\section{Acknowledgements}

We appreciate Mr Tomohiro Kuwazuru and Mr Akira Morita (Doshisha University) for their technical supports.

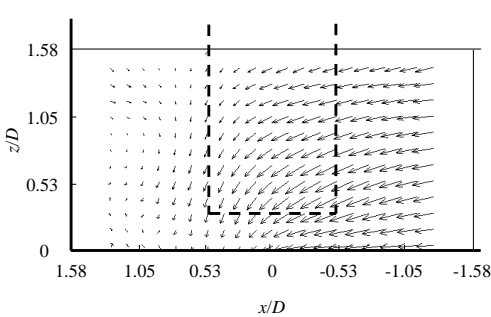

Fig. 15 Time-mean velocity vectors by $3 \mathrm{D}-\mathrm{PTV}$ at $y / D=-0.66$.

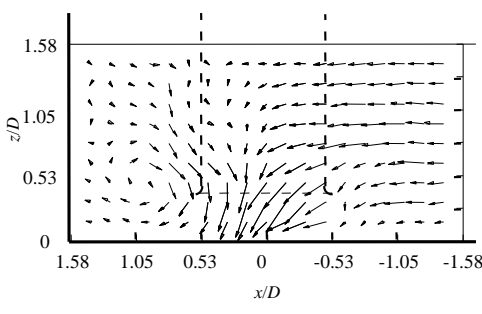

Fig. 16 Time-mean velocity vectors by UVP at $y / D=-0.63$

(Funaki et al., 2008).

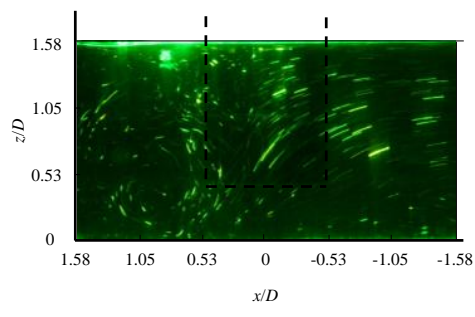

Fig. 17 Paths of tracer particles by a still camera on the $x-z$ plane at $y / D$ $=0.79$, with a shutter speed of $1 / 20 \mathrm{~s}$.

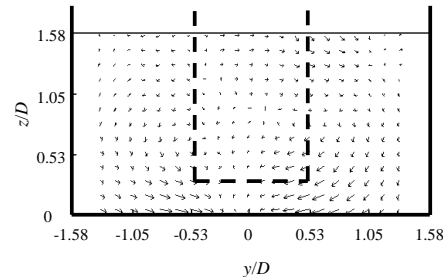

(a) $x / D=-1.32$.

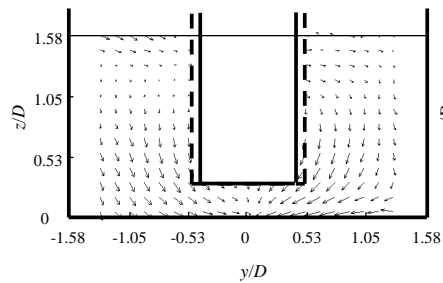

(d) $x / D=0.26$.

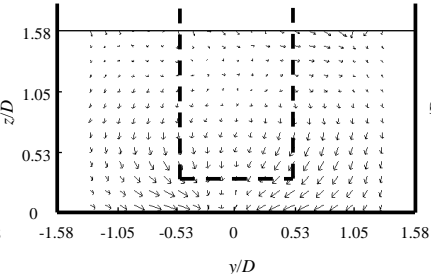

(b) $x / D=-0.79$.

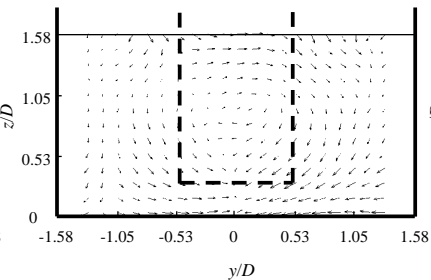

(e) $x / D=0.79$.

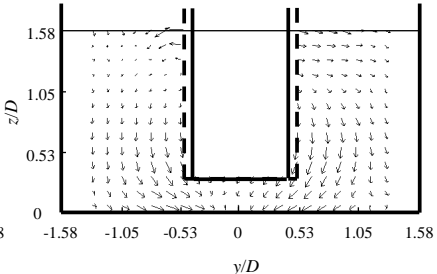

(c) $x / D=-0.26$.

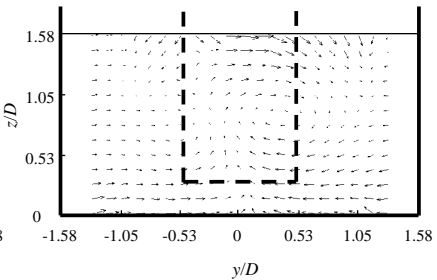

(f) $x / D=1.32$.

Fig. 18 Instantaneous velocity vectors by 3D-PTV. 


\section{References}

Adrian, R. J., Particle-imaging techniques for experimental fluid mechanics, Annu. Rev. Fluid Mech., Vol. 23 (1991), pp. $261-304$.

Chang, T. P. K., Watson, A. T. and Tatterson, G. B., Image processing of tracer particle motions as applied to mixing and turbulent flow - I, the technique, Chemical Engineering Science, Vol. 40, No. 2 (1985), pp. 269-275.

Doh, D. H., Kim, D. H., Cho, K. R., Cho, Y. B., Lee, W. J., Saga, T. and Kobayashi, T., Development of genetic algorithm based 3D-PTV technique, Jounal of Visualization, Vol. 5, No. 3 (2002), pp. 243-254.

Funaki, J., Neya, M., Hattori, M., Tanigawa, H. and Hirata, K., Flow measurements in a suction sump by UVP, Journal of Fluid Science and Technology, Vol. 3, No. 1 (2008), pp. 68-79.

Funaki, J., Shintani, A., Kawaguchi, R. and Hirata, K., Basic space structure of bubble-jet plume using consecutive 3D-PTV, Journal of Fluid Science and Technology, Vol. 4, No. 2 (2009), pp. $348-358$.

Goldberg, D. E., Optimal initial population size for binary-coded genetic algorithm, TCGA Report, No. 85001, University of Alabama (1985), pp. 1-13.

Hirata, K., Saito, K., Hattori, M., Nakatani, Y. and Funaki, J., Occurrence-time-ratio measurements on air entrainment in a suction sump, Journal of Fluid Science and Technology, Vol. 4, No. 1 (2009), pp. 47-61.

Hirata, K., Funaki, J., Kubota, Y., Inagaki, K., Taoka, M. and Saida, T., Occurrence condition of air entrainment into a vertical wet-pit pump, Journal of Fluid Science and Technology, Vol. 8, No. 3 (2013a), pp. $364-379$.

Hirata, K., Kawaguchi, R., Miki, T. and Funaki, J., 3D-PTV measuring on bubble and liquid velocities of a single bubble jet, Journal of Fluid Science and Technology, Vol. 8, No. 3 (2013b), pp. 423-435.

Nishino, K., Kasagi, N., Hirata, M. and Sata, Y., Three-dimensional flow velocimetry based on digital image processing, Transactions of the Japan Society of Mechanical Engineers, Series B, Vol. 55, No. 510 (1989), pp. 404-412 (in Japanese).

Tagomori, M., Flow pattern and air-entraining vortices in a suction sump, Turbomachinery, Vol. 7, No. 8 (1979), pp. 451-460 (in Japanese).

Takeda, Y., Velocity profile measurement by ultrasound Doppler shift method, International Journal of Heat and Fluid Flow, Vol. 7 (1986), pp. 313-318.

Takeda, Y., Fiscer, W. E., Kobayashi, K. and Takada, T., Spatial characteristics of dynamic properties of modulated wavy vortex flow in a rotating Couette system, Experiments in Fluids, Vol. 13 (1992), pp. 199-207.

Yamamoto, F. Tian, H. Z., Uemura, T. and Ohmi, K., Three-dimensional PTV based on binary cross-correlation method: algorithm of particle identification, Transactions of the Japan Society of Mechanical Engineers, Series B, Vol. 57, No. 543 (1991), pp. 3832-3836 (in Japanese). 